\title{
El portal "Tecnociencia": un sistema de Información Científica en Ciencia y Tecnología
}

\author{
Elena Fernández Sánchez \\ Centro de Información y Documentación Científica \\ Consejo Superior de Investigaciones Científicas (España)
}

\subsection{Resumen}

Se presenta el portal Tecnociencia como ejemplo de una iniciativa llevada a cabo por la administración para potenciar la difusión y la transferencia de resultados del trabajo científico y tecnológico llevado a cabo en España y en menor medida en el ámbito europeo, a todos los sectores de la sociedad. Se muestra la estructura del portal, los canales temáticos y los diferentes recursos de información referencial, contenidos singulares y servicios que se ofrecen a los usuarios.

Palabras clave: Información científico-técnica. Información al ciudadano. Administración pública. Sistemas de información. Internet. Portales.

\subsection{Abstract}

The Internet portal Tecnociencia is presented as an example of an initiative carried out by public administrations in order to enhance the dissemination and transfer of scientific and technological information to the whole society with a Spanish and, to a lesser extent, European scope. The structure of the website, its thematic channels and the different information resources that have been gathered - references and individual contents, and services - are presented.

Keywords: Scientific and technical information. Citizen information. Public administrations. Information systems. Internet portals.

\section{Introducción}

A través de la historia, el conocimiento ha ocupado siempre un lugar central tanto para el crecimiento económico de los países como para el bienestar social de sus ciudadanos. Sin embargo, no es hasta los últimos años en que surgen y se desarrollan las tecnologías de la información, cuando éste adquiere un papel absolutamente preponderante y se democratiza como nunca hasta ahora había ocurrido. La sociedad occidental ha experimentado una transformación de ser una sociedad eminentemente industrial a convertirse en la sociedad de la información y del conocimiento.

Scire. $10: 2$ (jul.-dic. 2004) 147-154. 
Todos los países han apostado por la sociedad del conocimiento como medio principal para el estímulo del crecimiento económico. Así, las administraciones públicas están desarrollando iniciativas con el objetivo de ofrecer a sus ciudadanos la información que ellas mismas elaboran y que tiene una importancia crucial para toda la sociedad, fomentando la competitividad en el sector empresarial y facilitando servicios a los ciudadanos que antes se realizaban a través de la famosa ventanilla, con la consiguiente pérdida de tiempo y de recursos económicos, tanto por parte del ciudadano como de las propias administraciones. De esta forma, se está creando una administración más transparente para todos y con un objetivo definido: Hacer llegar el conocimiento que genera a toda la sociedad.

En el caso concreto de la investigación científica, en la mayoría de los casos financiada con fondos públicos, los científicos deben rendir cuentas a la sociedad y devolverle los resultados que han conseguido. Pero no solo es necesario comunicar la ciencia por los canales habituales empleados por los científicos, como son la publicaciones periódicas, congresos o cualquier otra vía exclusiva para profesionales, sino también realizar su divulgación para toda la sociedad, con el fin de generar una imagen social de la investigación y del progreso científico diferente a la hasta ahora generada, y de trasladar directamente a los ciudadanos información de su interés.

En este contexto, Tecnociencia es un buen ejemplo de este tipo de iniciativas, en este caso fruto de la colaboración entre el Centro de Información y Documentación Científica CINDOC - perteneciente al Consejo Superior de Investigaciones Científicas (CSIC) - y el Ministerio de Ciencia y Tecnología para la elaboración de un portal especializado en información científica, donde se encuentran representadas las ciencias puras, experimentales, tecnológicas, médicas y de la vida, sociales y humanas.

El objetivo principal de Tecnociencia, es la recopilación, estructuración, difusión y divulgación de todos los recursos científicos y tecnológicos pertenecientes a nuestro país, con el objetivo de que cualquier ciudadano pueda acceder a ellos y de convertirse en sitio de referencia de la información científica. Así mismo, a través de su servicio de información, pretende ser una ventanilla directa para atender todas las consultas realizadas por los usuarios que necesiten información sobre temas relacionados con la investigación científica y técnica desarrollada en España. Su URL es: <http://www.tecnociencia.es>.

\section{Contenidos}

\subsection{Información referencial}

El núcleo central de Tecnociencia es un directorio de más de 30.000 recursos de información estructurados en los veinticuatro canales temáticos siguientes: 
Agricultura; Biología Molecular Celular y Genética; Biología Vegetal Animal y Ecología; Ciencia y Tecnología de los Alimentos; Ciencia y Tecnología de los Materiales; Ciencias de la Computación y Tecnología Informática; Ciencias de la Tierra; Ciencias Sociales -Comunicación, Documentación, Geografía, Sociología, Política, Urbanismo -; Derecho; Economía; Farmacología y Farmacia; Fisiología y Filosofía; Física y Ciencias del Espacio; Ganadería y Pesca; Historia del Arte; Ingeniería Civil, Transportes, Construcción y Arquitectura; Ingeniería Industrial, Mecánica, Naval, Aeronáutica y Medioambiental; Matemáticas; Medicina; Psicología y Ciencias de la Educación; Química; Tecnología de las Comunicaciones; Ingeniería Eléctrica, Electrónica y Automática; y Tecnología Química.

Estos canales están distribuidos en las siguientes secciones: organizaciones —organismos de la administración, instituciones académicas y de investigación, asociaciones y fundaciones, etc. - , agenda - cursos y congresos - , proyectos de investigación, recursos web - documentos y revistas electrónicas, páginas temáticas, bases de datos, etc. - y noticias científicas y de prensa.

Una vez identificados los recursos, se realiza un análisis documental para obtener los descriptores, un resumen indicando los aspectos más sobresalientes del recurso, topónimos (en determinados casos), dirección electrónica de contacto y dirección web. De esta forma, el usuario visualiza la referencia del recurso, tiene una idea bastante aproximada de lo que va a encontrar y en definitiva si le interesa o no.

Dentro de las secciones señaladas, las noticias de prensa, científicas y los documentos se presentan a texto completo. Además, los históricos de las noticias constituyen una magnífica fuente de información para detectar el pulso científico y técnico de lo ocurrido desde el año 2001, año del comienzo del proyecto, hasta la actualidad tanto en el ámbito nacional como internacional.

\subsection{Distribución de los recursos por canales temáticos}

El número total de recursos contenidos en PortalTecnociencia hasta Julio de 2002, es de 36.059. Su distribución por canales temáticos se presenta en la figura 1. La figura 2 muestra la distribución por tipología del recurso.

\subsection{Contenidos singulares}

Se han desarrollado una serie de contenidos que singularizan al portal en base a la experiencia adquirida con otros portales elaborados por el CINDOC. A continuación se describen en que consisten estos contenidos:

1. Especiales CINDOC. Son documentos monográficos, elaborados por el equipo de documentación del CINDOC con periodicidad aproximadamente mensual, en los que se procura recoger, aunque no es condición necesaria, temas 


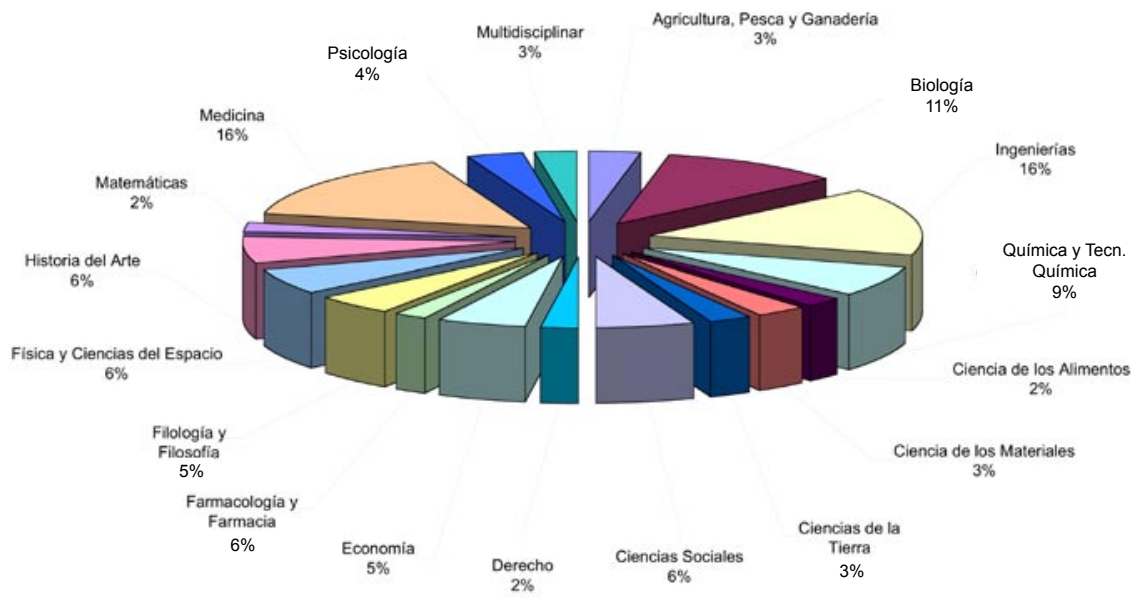

Fig. 1. Distribución por canales temáticos de los recursos del portal Tecnociencia

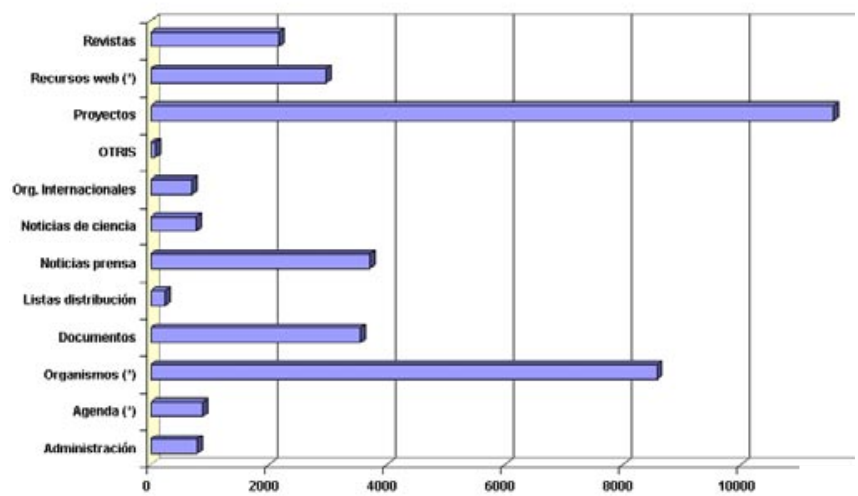

Fig. 2. Distribución por tipología de los recursos del portal Tecnociencia

que en ese momento gocen de plena actualidad. La característica fundamental de estos especiales es la gran cantidad de referencias que realizan a fuentes externas, estructuradas en diferentes temas: legislación, bases de datos, documentos emitidos por organizaciones de reconocido prestigio, revistas científicas, proyectos de investigación que se están desarrollando sobre el tema, etc. De esta forma, cualquier usuario interesado en la materia tratada, puede profundizar y obtener la información más actualizada existente en la red. 
Fig. 3. Vistas de la página de inicio, suscripción a boletines $y$ contenidos especiales (de arriba a abajo e izquierda a derecha)

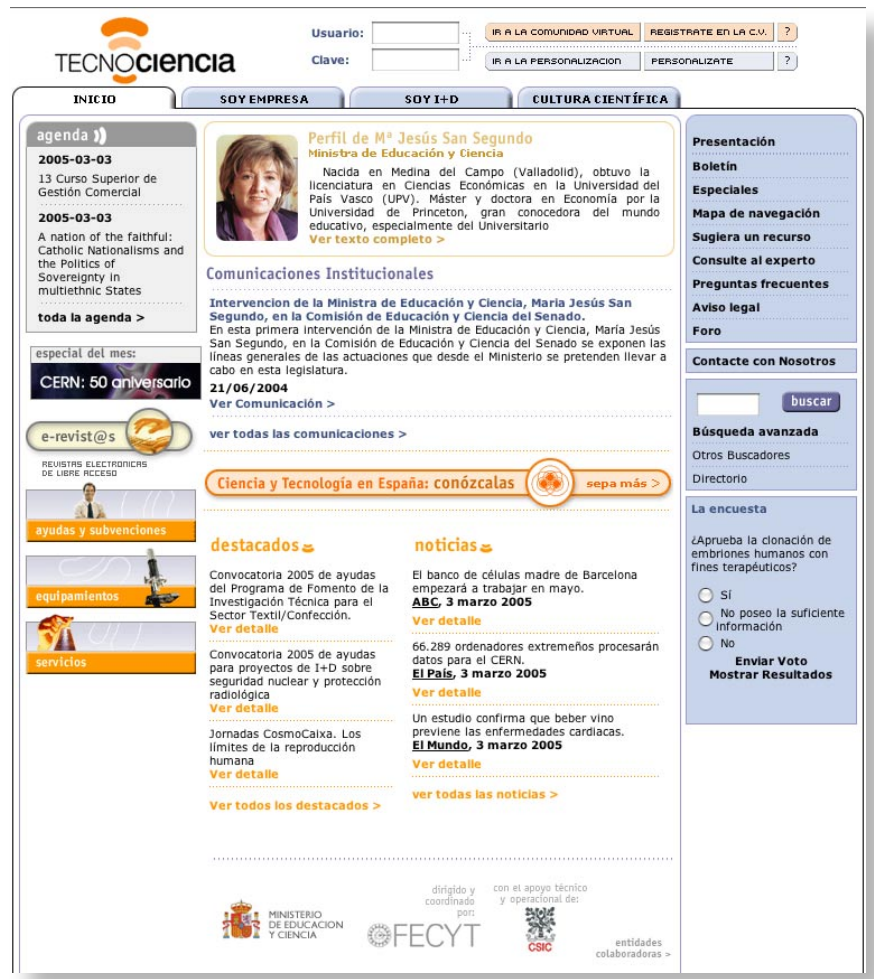
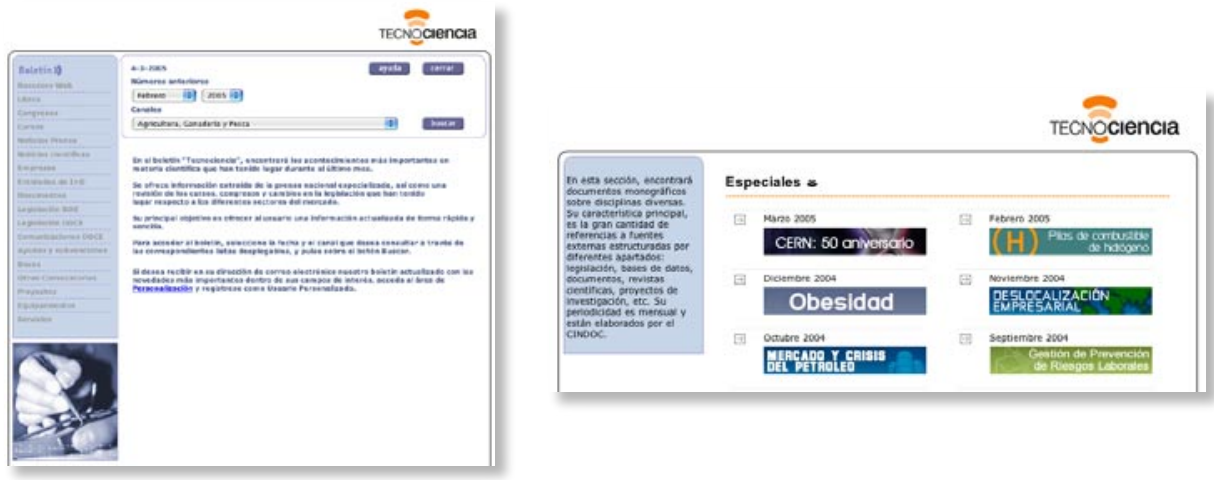

Scire. $10: 2$ (jul.-dic. 2004) 147-154. 
2. Boletín Tecnociencia. Consiste en un boletín electrónico de edición mensual que constituye una alerta científica-técnica y legal e incluye también información sobre ayudas y subvenciones, becas, convocatorias de proyectos, etc. En cada boletín se recogen los eventos más importantes que han tenido lugar durante el último mes, así como los que se van a desarrollar durante el mes próximo. El boletín está estructurado por canales, de forma que el usuario puede acceder directamente al canal o canales de su interés, y obtener una información rápida y completa de todos los eventos que se han desarrollado o se van desarrollar en esa disciplina. Los recursos que se recogen por canales en los boletines son los siguientes: noticias de prensa, noticias científicas, congresos, cursos, ayudas y subvenciones, legislación BOE, becas, comunicados DOCE y legislación DOCE. Así mismo, dispone de una sección donde se recomiendan sitios web y libros. En un futuro, se contempla la posibilidad de enviar el boletín por e-mail a los usuarios que lo deseen.

3. Ventana de la Ciencia. Este apartado está especialmente dirigido a la comunidad docente de primaria y secundaria. Sus contenidos los realiza el Instituto de Matemáticas y Física Fundamental del Consejo Superior de Investigaciones Científicas, y están supervisado por un equipo de profesores especializados en divulgación científica para los más pequeños.

\section{Servicios}

Los principales servicios que se ofrecen son los siguientes:

1. Buzón del usuario. A través de este servicio, PortalTecnociencia ofrece un sistema de información rápido y eficaz donde el usuario puede formular sus dudas o enviar sus sugerencias. PortalTecnociencia puede encontrar la información en un plazo máximo de 48 horas.

2. Sugerir un recurso. A través de un sencillo formulario, cualquier usuario puede introducir los recursos que estime conveniente. Estas sugerencias son comprobadas por los documentalistas, y posteriormente se incorporan al portal.

3. Sistema de búsqueda. El acceso a la información se puede realizar a través de bases de datos o por navegación a través de directorios. La búsqueda en bases de datos permite buscar por canal, tipo de recurso, topónimo (para algunos tipos de recursos) y fecha.

\section{Organización del trabajo}

Para llevar a cabo la creación del portal, el trabajo se distribuyó entre los tres equipos responsables de las tareas de documentación, diseño e informática. Estos equipos, están coordinados por el jefe el proyecto. En la figura 4 se muestra el esquema de la organización. 
Las tareas a desarrollar por el equipo documental han sido el diseño de contenidos y estructura de los campos de las bases de datos; la identificación, evaluación y selección de los recursos en la red; el análisis documental de los recursos para optimizar la recuperación de los mismos - resumen, descriptores, topónimos, clasificación, direcciones, URL -; la elaboración de contenidos singulares - especiales CINDOC, Boletín electrónico, Ventana de la Ciencia (contenidos elaborados por el Instituto de Matemáticas y Física Aplicada del CSIC); la planificación y coordinación del diseño del portal; la difusión del portal; y la atención al servicio de atención al usuario.

Para la realización de todas estas tareas y teniendo en cuenta el carácter multidisciplinar del portal, se nombraron cinco coordinadores que se hicieron responsables de los contenidos por grandes áreas: Ciencias Puras, Tecnología, Ciencias de la Vida, Ciencias Sociales y Ciencias Humanas.

\section{Diseño del portal}

La elaboración del diseño del portal se ha realizado en estrecha colaboración entre el equipo documental e informático. El diseño permite acceder a todos los contenidos desde la primera página. Las páginas son todas dinámicas y se han

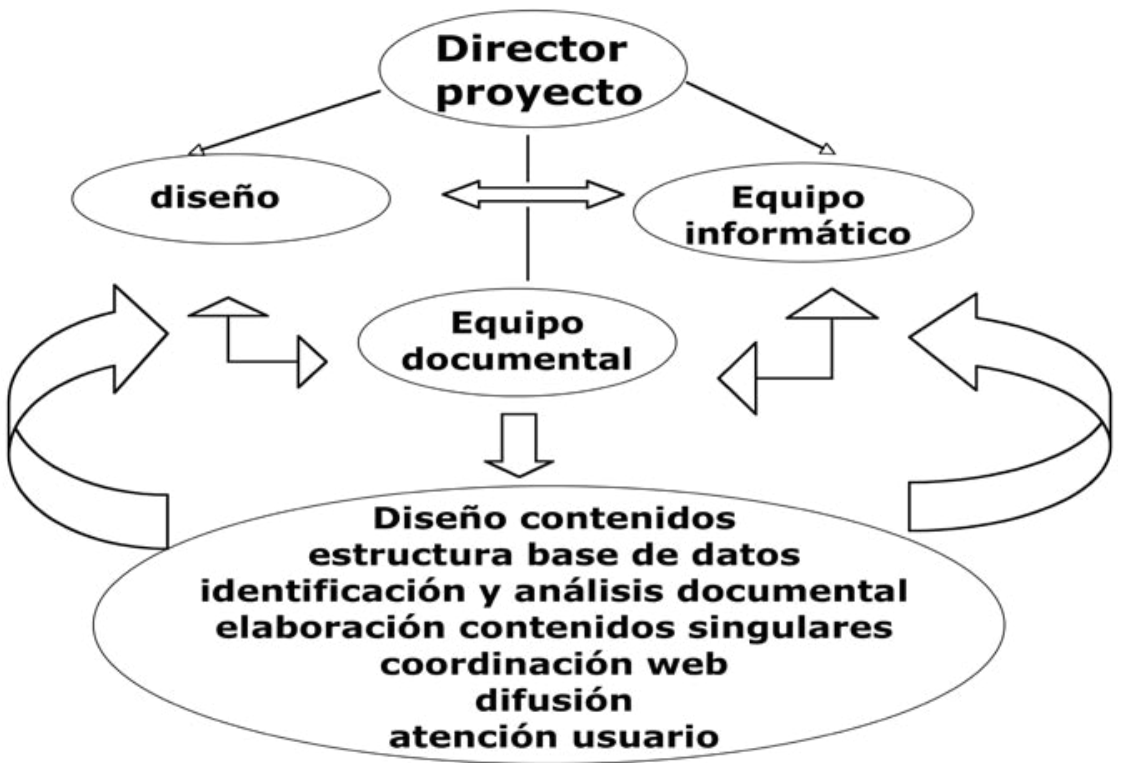

Fig. 4. Organización del equipo de trabajo

Scire. $10: 2$ (jul.-dic. 2004) 147-154. 
desarrollado con el editor de páginas Dreamweaver, y también cuenta con elementos de Flash y Fireworks para diversas animaciones. Otros elementos multimedia con los que cuenta son archivos de vídeo. Se ha procurado que el tamaño de los archivos sean pequeños con objeto de optimizar el tiempo de conexión. El diseño está optimizado para Explorer a partir de la versión 5 y para Netscape a partir de la 6 y para una resolución de pantalla mínima de 800 x 600. Se han introducido metaetiquetas en cada una de las páginas. Actualmente se esté rediseñando el web para conferirle todos los criterios de usabilidad.

\section{Conclusiones finales}

Este proyecto se ha realizado gracias a financiación de la Fundación Ciencia y Tecnología (FECYT). Lo que pretendemos en este artículo es, por un lado, presentar el portal a la comunidad de internautas, haciendo énfasis en que no está dirigido solo a la comunidad científica y técnica, sino a un sector mucho más amplio de todo el mundo hispano-parlante representado por estudiantes y profesionales de todas las disciplinas científicas y técnicas. Finalmente, a través del sistema de información del usuario, se pretende abrir una "ventana de la ciencia" donde los usuarios puedan formular sus dudas y preguntas sobre cualquier tema de su interés relacionado con la Ciencia y la Técnica, así como animarles a que enviar sus colaboraciones y trabajos para su publicación en el web. En definitiva, se busca expresamente la colaboración de los usuarios.

Todo el equipo de PortalTecnociencia que ha contribuido a la elaboración del portal, desea que el proyecto cumpla los objetivos para los que fue creado, y espera sugerencias y comentarios para intentar mejorar y conseguir un producto de calidad y útil para todos. 